\title{
Contribución de la innovación deportiva al crecimiento económico europeo
}

\section{Contribution of sport innovation the european economic growth}

\author{
Silva Piñeiro, R. \\ Doctor en Ciencias del Deporte. Universidad de Vigo, España
}

\begin{abstract}
Resumen: Las Instituciones Europeas han reconocido la destacada contribución que el sector del deporte está teniendo en la creación de empleo, el desarrollo económico local y en los flujos intersectoriales, convirtiéndose en uno de los pilares que permitan ayudar a superar las dificultades actuales gracias a la gran resistencia que ha demostrado en el entorno de crisis económica que venimos arrastrando. El deporte puede consolidar el impacto socio-económico que ha alcanzado, e incluso mejorarlo, a través de servicios y productos innovadores, que en cualquier caso deberán atender sobre todo al nivel regional, clave del éxito de cualquier conocimiento.

Palabras clave: Desarrollo económico, innovación, tecnología, deporte, financiación, regiones.
\end{abstract}

Abstract: The European institutions have recognized the outstanding contribution to the sport sector is having on job creation, local economic development and intersectoral flows, becoming one of the pillars that allow help overcome the current difficulties due to the high resistance which has been shown in the environment of economic crisis we have been dragging. Sport can strengthen the socio-economic impact it has had, and even improve, through innovative services and products, which in any case must attend especially at the regional level, the key to success of any knowledge.

Keywords: Economic development, innovation, technology, sports, finance, regions.

\section{Dimensión del deporte en la economía y el empleo en Europa}

En general, Europa está asimilando, con cierta demora, la importancia del deporte en el crecimiento y la innovación. Los organismos internacionales tienen hoy claro que el deporte puede contribuir a superar las dificultades económicas actuales de la UE, ya que es un sector en desarrollo y que ha mostrado una resistencia excepcional durante la crisis económica ${ }^{1}$. Las autoridades europeas han reconocido la importante aportación del deporte a la economía de la UE, en particular en materia de creación de puestos de trabajo y desarrollo económico local, junto con los efectos multiplicadores en otros sectores ${ }^{2}$.

En 2012 se presentaban los resultados sobre la contribución del deporte al crecimiento económico y el empleo en la Unión Europea, destacando el aumento de cuatro subsectores de la industria del deporte: el turismo activo, gracias a la fuerte demanda interna en países como Alemania y Suecia, los turistas internacionales en Austria e Italia, y el apoyo importante en el sector de la hostelería; el fitness, sobre todo en el noroeste europeo, Suecia y Holanda, por la gran deman$\mathrm{da}$ comercial de actividades deportivas indoor; los medios de comunicación, aprovechando el efecto tractor del fútbol profesional y comunicación en países como el Reino Unido; y la educación deportiva, elemento transversal de la economía en todos los países, con mayor incidencia en Dinamarca, Estonia y Letonia ${ }^{3}$.

Hace una década se estimaba la importancia del deporte en el PIB europeo en el $1,6 \%{ }^{4}$, aunque ante la disparidad de cifras que aparecían, se hizo evidente la necesidad de una

Dirección para correspondencia [Correspodence address]: Roberto Silva Piñeiro. Universidad de Vigo (España). E-mail: roberto.silva@uvigo.es definición del deporte relacionado con la economía 5 . Actualmente el deporte recoge en su definición amplia todos los productos y servicios necesarios para el deporte, y todos aquellos bienes o servicios que tienen relación indirecta pero no son necesarios para hacerlo ${ }^{6}$.

En 2014 generó el 2,12\% del empleo en la UE, llegando a los 4,46 millones de trabajadores, y superando el valor añadido bruto del deporte (1,76\%). De ellos, en Alemania se concentra el $27 \%$ de todos los empleados del deporte en la UE (1,15 millones), seguido de Reino Unido (610.000) y Francia (410.000). Los efectos directos en el empleo ascendieron a 41460.888 de personas, que sumando los indirectos alcanzaron los 71378.671 trabajadores (3,51\%). En Espańa, el empleo generado fue menor, contabilizándose 336.177 personas $(1,77 \%)$.

El sector del deporte se ha convertido en una industria de trabajo intensivo, pues su crecimiento previsto conduce a que se produzca un empleo adicional, participando del empleo total de forma superior a su participación en el valor añadido ${ }^{3}$. Se estima que la producción y servicios de deporte crecerán más rápido en los países de ingresos más bajos que en los más ricos, contribuyendo a la convergencia económica dentro de la UE. Mientras en España el nivel del Valor Añadido Bruto (VAB) del deporte es un 50\% más bajo que en la $\mathrm{UE}$, el deporte europeo aporta en términos de producción e ingresos seis veces más de lo que refieren las estadísticas oficiales, y junto con sus multiplicadores, alcanzan el 2,98\% del VAB. Su participación en la economía es comparable a la agricultura, silvicultura o pesca, y casi 2,5 veces más grande que la minería y la explotación de canteras, alcanzando los 174 mil millones de $€$, de los cuales el $73 \%$ proviene del 
sector de servicios ${ }^{7}$. Actualizando anteriores enfoques ${ }^{8,9}$ se identificaron aquellos sectores clave para el crecimiento y los resultados muestran que "productos alimenticios y bebidas", "construcción", y "transporte" son estratégicos en todos los países. Igualmente hay una importante concentración en el "comercio", "hoteles y restauración", e "intermediación financiera y seguros".

Tabla 1. VAB a la economía en miles de millones de $€^{10}$

\begin{tabular}{llc}
\hline Construcción & & 3,2 \\
\hline Transporte & Terrestre & 5,8 \\
\hline Manufactura & Impresos y soportes grabados & 3,4 \\
& Fabricación de equipamiento deportivos & 3,4 \\
& Fabricación de embarcaciones y transportes & 1,5 \\
& Prendas de vestir y pieles & 2,9 \\
& Vehículos de motor y remolques & 1,4 \\
& Cuero y productos de cuero & 1,3 \\
& Maquinaria & 1,3 \\
& Textiles & 1,2 \\
& Productos metálicos & 1,2 \\
& Sustancias químicas y fibras & 1,1 \\
& Caucho y materias plásticas & 0,9 \\
& Productos alimenticios y bebidas & 0,6 \\
& Sistemas de medición & 0,4 \\
\hline Servicios & Servicios recreativos, culturales y deportivos & 52,7 \\
& Educación & 22,2 \\
& Hoteles y restaurantes & 17,4 \\
& Servicios de salud y asistencia social & 8,8 \\
& Comercio minorista & 7,5 \\
\hline & &
\end{tabular}

El VAB en la UE más alto corresponde a los servicios deportivos, culturales y recreativos, y los servicios educativos, que se generan principalmente en Alemania, Reino Unido, Francia, Italia y Austria. Para un correcto balance económico hay que considerar la importancia de los efectos multiplicadores sectoriales, que describen la interconexión de un sector con el resto.

En el conjunto de países de la UE los multiplicadores más altos se encuentran en la construcción, alimentación $\&$ bebidas, y sectores relacionados con el turismo (hostelería y transporte). Algunos países están aprovechando de forma extraordinaria las ventajas de especialización del deporte, como multiplicadores específicos para el fomento del crecimiento, como en el Reino Unido (deporte profesional y apuestas), en Austria (turismo alpino), o en el norte de Europa (educación). En España los valores de los multiplicadores son semejantes, con alguna diferencia favorable en el sector de la construcción, alimentación y bebidas, e industria textil.

Tabla 2. Ranking comparativo sobre VAB a la economía en millones de $€^{3}$

\begin{tabular}{ccccc}
\hline UE & \multicolumn{2}{c}{ ESPAŃA } \\
\hline Servicios deportivos, culturales y recreativos & $52.718,52$ & 1 & $2.821,94$ & Servicios sociales y de salud \\
Servicios educativos & $22.220,68$ & 2 & $2.767,07$ & Servicios educativos \\
Servicios de hostelería y restauración & $17.427,68$ & 3 & $1.114,30$ & Construcción \\
Servicios sociales y de salud & $8.896,62$ & 4 & 477,56 & Transporte terrestre \\
Comercio minorista & $7.556,14$ & 5 & 415,27 & Servicios de hostelería y restauración \\
Transporte terrestre & $5.816,82$ & 6 & 280,69 & Servicios deportivos, culturales y recreativos \\
\hline
\end{tabular}


En la UE estamos asistiendo al reconocimiento de la potencialidad del deporte en este sentido. Las actividades deportivas requieren importantes inversiones en estadios, edificios e infraestructuras, en concreto, la renovación de una serie de estadios de fútbol para la Eurocopa de fútbol de 2016 en Francia requerirá inversiones de 2 mil millones €, mientras que los proyectos de construcción para los Juegos Olímpicos de Londres en 2012 ascendieron a 2.900 millones $€$. Igualmente el turismo deportivo está contribuyendo a este fenómeno, pues genera en la UE entre 12 y 15 millones de viajes internacionales cada ańo, y se espera que la tasa de crecimiento sea del $6 \%$ por año durante los próximos dos años.

Además, un efecto indirecto de los eventos es que los principales destinos turísticos están desarrollando productos de deporte recreativo para atraer más turismo. La educación por su parte, principalmente en países nórdicos, tiene un efecto multiplicador transversal, aunque más bajo ya que no necesita muchos bienes intermedios en comparación con el salario.

Tabla 3. Media del Multiplicador específico sectorial en la UE. PIB ponderado ${ }^{3}$

\begin{tabular}{lcc}
\hline & UE & ESP \\
\hline Construcción & 2,14 & 2,48 \\
Alimentación y bebidas & 2,06 & 2,42 \\
Seguros & 2,00 & 1,93 \\
Transporte aéreo & 1,93 & 1,97 \\
Transporte terrestre & 1,83 & 1,93 \\
Servicios de hostelería y restauración & 1,82 & 1,80 \\
Servicios deportivos, culturales y recreativos & 1,76 & 1,71 \\
Textiles & 1,65 & 1,91 \\
Servicios financieros & 1,61 & 1,34 \\
Servicios sociales y salud & 1,53 & 1,57 \\
Servicios educativos & 1,34 & 1,23 \\
\hline
\end{tabular}

Es fundamental que el sector deportivo se apuntale como tractor económico, con más acciones concretas hacia el deporte e industrias relacionadas y aportaciones de todos los sectores económicos relacionados, el mundo académico y las asociaciones ${ }^{11}$, similares a las recogidas de los organismos eu$\operatorname{ropeos}^{10}$ :

1. Maximizar la eficiencia de la inversión en infraestructura deportiva.

2. Fomentar la competitividad industrial del deporte, aprovechando la estrecha colaboración con la industria textil y electrónica, protegiendo más los derechos de propiedad intelectual, y facilitando el comercio e inversión entre países de la UE.
3. Desarrollar estudios sobre la eficacia y la sostenibilidad de los eventos deportivos y sobre las nuevas tendencias/oportunidades a considerar por la industria del deporte.

4. Aprovechar plenamente las posibilidades de financiación europea.

5. Habilitar la maximización de los efectos indirectos del deporte en el crecimiento y el empleo (Considerando que cada nuevo trabajo genera 0,65 nuevos puestos de trabajo en industrias afines).

6. Crear organismos específicos regionales para facilitar la organización de eventos deportivos y facilitar las actividades turísticas relacionadas con el deporte y el turismo en grandes acontecimientos deportivos.

7. Otorgar al deporte un IVA específico.

\section{El fenómeno de la innovación deportiva en el mundo}

Entre dos de los motores económicos mundiales, Estados Unidos y Europa, la cultura de innovación presenta una notable distancia, lo que repercute en su economía. En Estados Unidos, el IBE es el primer índice multifactorial utilizado para identificar la capacidad de innovación de las $100 \mathrm{ma}-$ yores empresas internacionales analizadas según 35 criterios, que no responden a fuentes tradicionales sino a las nuevas formas de innovar, pues hoy en día las patentes, los derechos de autor y las marcas comerciales ya no funcionan con la misma eficacia para proteger el ingenio que inventores y empresas aportan a sus productos ${ }^{12}$.

Tanto en este índice y en otros, como Fast Company ${ }^{13}$ o Kate Rockwood ${ }^{14}$, la empresa de calzado y artículos deportivos Nike está por delante de los gigantes tecnológicos como Google, Microsoft o Amazon. Las razones de este reconocimiento responden al enfoque de su negocio a una forma de hacer actividad física virtualmente, desde la incorporación del iPod a principio de los años 2000 a la comercialización reciente de Fuelband, siempre a la vanguardia de la wearable computing. Entre otras proyectos innovadores destacaron durante 2014 la National Basket Association (NBA), que ha puesto en funcionamiento una estrategia slam-dunk para involucrar a los aficionados a través de sus productos digitales y comunicación; Qcue, un sistema de búsqueda y gestión de entradas a eventos deportivos; AlterG, que ofrece unas cintas de correr antigravedad; Babolat, con la fabricación de raquetas de tenis con sensores de datos integrados en el mango; o GE Salud, mediante equipos portátiles de resonancia magnética para el análisis de lesiones en la cancha.

Podríamos mencionar también a SportVU, por la instalación de cámaras de pista y cuantificadores durante los partidos, versátil para los propios equipos deportivos, en la plataforma web o a través de la TV; a IBM, por el software SlamTracker de captura los datos de los sensores de movi- 
miento en los partidos de tenis, análisis visual y predicción del resultado; o Replay Technologies, por su sistema de cámaras de alta velocidad para acciones deportivas, que se pueden visualizar en $360^{\circ}$ en red o en los propios estadios. Del mismo modo destacaron Soccket, un balón de fútbol inventado por Jessica Matthews y Julia Silverman capaz de generar en 30 'de juego cerca de tres horas de energía para lámparas led, y Pulse, una cuerda para saltar que puede generar cinco veces la electricidad de la Soccket.

Otras innovaciones notorias han sido lanzadas por la cadena CrossFit, al aumentar los gimnasios de su red en alianza con Reebok y la cadena televisiva ESPN para transmitir sus eventos deportivos; Ciclo 3-Soul, al introducir bicicleta estáticas en entrenamientos que mezclan yoga, fitness y rock $\&$ roll; FITiST, mediante una novedosa plataforma de información y reserva de clases en centros de fitness a precios reducidos e incluso gratis; o Elliptigo, con una colección de bicicletas híbridas con un mínimo impacto articular.

En Europa, es interesante el caso de Holanda, que en 2006 creaba la Plataforma InnoSportsNL como un organismo intermedio para poner en contacto el deporte, el conocimiento y el mundo empresarial ${ }^{15}$. La finalidad no sólo estaba centrada en los mejores resultados del deporte de alto nivel y en animar a la gente a ser más activos y participar en los deportes, sino también en generar negocio. Una decisión excelente, pues el tiempo les está dando la razón, fue la de crear el primer laboratorio de campo en Brainport-Eindhoven, considerada entre las regiones más avanzadas del mundo para la investigación e innovación, para probar nuevas ideas, conceptos, tecnologías y productos relacionados con el deporte recreativo y de competición.

Además, como ejemplo para otros proyectos, esta iniciativa se financió por fondos europeos, concretamente en el marco del programa Interreg IVB Noroeste de Europa a través del Proyecto Profit, cuyo principal objetivo es estimular la innovación y la creación de nuevas empresas en el sector del deporte mediante el desarrollo de una red de laboratorios de campo centrados en el deporte de la $\mathrm{UE}^{16}$. Estos laboratorios están ubicados en Eindhoven y Delft (Holanda), Kortrijk (Bélgica) y Sheffield (UK), donde los ciudadanos pueden participar en diversas actividades deportivas innovadoras. Holanda además ha conseguido poner en marcha otros laboratorios, conocidos como InnoSportLabs, entre ellos el International Sailing Centre Den Haag y el InnoSportLab Gimnasia, que trabajan en colaboración con otros centros tecnológicos y deportivos, cada uno en una determinada rama del deporte. En esta línea de colaboración, la Organización Holandesa para la Investigación Científica (NWO) y la Organización Holandesa para la Investigación en Salud y Cuidado Innovación (ZonMW), están trabajando en el programa integral de investigación en ciencias del deporte, en el que también participarían los institutos de formación profesional superior.
Como muestra de la buena acogida que los proyectos deportivos empresariales están teniendo este país referirnos a que, teniendo en cuenta el aumento de la inversión total, en 2008 el gobierno holandés cofinanció el 50\% de estas iniciativas, mientras tan sólo dos años más tarde, fueron patrocinados por la empresa privada el $85 \%$.

Holanda está volcando en el deporte un esfuerzo tecnológico y políticas de promoción deportiva y vida saludable que están reflejándose en innovaciones concretas, como sistemas avanzados de iluminación y comunicaciones en los estadios de fútbol, incorporación a las salas de baloncesto de sensores de pisada especiales y sistemas de visionado de imágenes instantáneo, o implementación en centros de gimnasia de anillas inteligentes y mecanismos de retroalimentación neurológica. Particularmente varias empresas holandesas son líderes mundiales en instalaciones de hielo y patinaje, pero también en superficies artificiales y césped artificial, equipamiento de gimnasio o nutrición deportiva. Y conviene recordar que este país es el referente mundial de la hípica, con una proyección hacia los 450.000 caballos, 6.600 empresas, 1.100 instalaciones ecuestres, 12.500 empleos y 7.424 eventos anuales.

Posibilidades de financiar la innovación deportiva en Europa

El reto de la financiación está ocupando a las autoridades europeas desde un clima de austeridad y consolidación fiscal, planteándose un presupuesto para el crecimiento en los próximos ańos. Así, se han presentado mejoras de las partidas destinadas a investigación e innovación, educación y desarrollo de las PYME, que descansan en el marco estratégico común para la investigación, la innovación y el desarrollo tecnológico ${ }^{10}$. El sector del deporte está dejándose ver con más intensidad en el debate de la economía y la innovación, y a principios de 2014 se planteaba el Segundo Plan de Trabajo de la Unión Europea para el Deporte 2014-2017, en el que se destacó la contribución del deporte a los objetivos globales de la Estrategia Europa $2020^{17}$.

Todas las correlaciones sectoriales serán necesarias, aunque si la UE quiere seguir compitiendo con otras economías tiene que solucionar la ruptura de innovación detectada, pues está quedándose atrás de Japón y Estados Unidos en el número de patentes, exportaciones de alta tecnología, o en porcentaje del gasto del PIB en I+D. El desafío no es sólo promover una mayor inversión en $\mathrm{I}+\mathrm{D}$ y mejorar la conversión del conocimiento científico en alta tecnología, sino que se extienda a los sectores tradicionales y emergentes, y a otras formas de hacer. Los primeros pasos han sido la creación del Consejo Europeo de Investigación, la reorganización de los instrumentos de financiación de la UE para la investigación e innovación, y de una reducción de los procedimientos administrativos. En cuanto al esfuerzo económico, el presupuesto de la UE para el período 2014-2020 asciende a 11025.000 millones $€$, de los que está previsto invertir directamente 80 mil millones $€$ en 
investigación e innovación, de los que 15.210 millones $€$ serán para de educación, juventud y deporte complementados con Fondos Estructurales ${ }^{18}$.

Si es importante reservar financiación, también lo es la eficiencia de los instrumentos que articulan la política regional de innovación e I+D. La UE ha utilizado sobre todo los Fondos Estructurales (Fondo Europeo de Desarrollo RegionalFEDER y el Fondo Social Europeo-FSE), el Programa Marco de Investigación y Desarrollo Tecnológico, y las Acciones Innovadoras (dentro de los FEDER), que incluyen entre otras las Estrategias Regionales de Innovación (RIS), los Proyectos de Estrategia Regional de Innovación y Transferencia Tecnológica (RITTS), y los Planes Tecnológicos Regionales (RTP). En la actualidad, otras posibilidades de financiación pueden encontrarse en el programa para la competitividad de las empresas COSME ${ }^{19}$, o en el Programa Erasmus $+^{20}$, y en el futuro se pretende facilitar el acceso a fuentes de financiación innovadoras, mejorar la contratación pública para la innovación, y potenciar el capital riesgo, de cara también a la auto-sostenibilidad financiera.

En esta cuestión y con referencias hacia el deporte, ocupa un lugar destacado el Programa Marco para la Investigación e Innovación en la UE, denominado Programa Horizonte 2020, que intenta incentivar la creación y el desarrollo de agrupaciones empresariales, tanto intersectoriales como transfronterizas donde participen las administraciones locales, universidades, centros de investigación y organismos representativos de los sectores productivos y del deporte, especializados en $\mathrm{I}+\mathrm{D}$, transferencia de tecnologías y soluciones innovadoras para el deporte, que contribuyan a la actividad económica en su conjunto, facilitando la aparición de efectos multiplicadores ${ }^{21}$. El deporte tiene un incentivo intrínseco e inherente que empuja a quienes lo practican a mejorar constantemente sus prestaciones y que atrae un volumen considerable de recursos económicos mediante el patrocinio y la venta de derechos de retransmisión, consiguiendo así capacidad para invertir en el desarrollo de equipos y materiales innovadores que pueden transformarse en ventajas competitivas. Esta combinación explica que el sector del deporte se caracterice por oleadas rápidas y constantes de innovación ${ }^{22}$, y el Programa Horizonte 2020 reconoce la importancia clave de las inversiones estratégicas en tecnología, también en el ámbito del deporte ${ }^{23}$.
La importancia de las regiones europeas en la innovación

Las innovaciones han convertido a la tecnología deportiva un sector líder en algunos ámbitos de las ciencias aplicadas, como la tecnología textil, la mecánica del movimiento humano, los nuevos materiales, los sensores, o el diseño orientado al ser humano. La categoría más evidente de la innovación deportiva es la que permite a los deportistas alcanzar mejores resultados, bien a través de la aplicación de nuevos materiales (nano-componentes, aleaciones o textiles técnicos), mejor equipamiento (calzado, bicicletas o raquetas), o mediante una perfeccionada nutrición. Otra categoría se refiere a la necesidad de garantizar que las normas se apliquen correctamente antes, durante y después los eventos deportivos (clasificaciones, arbitraje, controles, etc.). La última categoría descansa en el deseo de ofrecer "in situ" mayor comodidad y seguridad al espectador (asientos ergonómicos, microclimas, sistemas de seguridad, etc.), y mejorar la experiencia que comparte cualquier otro espectador allí donde se encuentre a través de nuevas formas de comunicación (entornos inalámbricos y pantallas gigantes en los estadios, repeticiones inmediatas, portales de estadística, etc.).

Para que el deporte pueda consolidar su relevancia socioeconómica tendrá que conseguir servicios y productos innovadores, que deberán atender a todos los niveles territoriales, especialmente al regional, pues en su dinamismo está la clave para que cualquier conocimiento se convierta en una exitosa innovación ${ }^{24}$. En este sentido, España fue un modelo de desarrollo de políticas regionales de innovación gracias a la estructura de competencias autonómicas en investigación e innovación coordinadas con las políticas estatales, en un modelo multinivel, en el que conviven diferentes niveles administrativos internacionales, nacionales, regionales y locales, que han evolucionado junto al proceso de regionalización ${ }^{23}$.

En la actualidad, la globalización minimiza el control estatal, por lo que cada región está trabajando sobre todo para captar recursos productivos más móviles y adaptar las políticas al aumento de la competitividad y la eficacia productiva. Cada día con mayor regularidad asistimos a una interacción entre proyectos regionales y locales, incentivados por su parte a través de directrices europeas que exigen la implicación de diferentes países. España y Bélgica son los países de la UE en los que tienen mayor importancia las medidas de apoyo regionales, al contrario de Francia o Reino Unido.

Tabla 4. Porcentaje de las medidas de apoyo a las empresas según niveles de gobierno ${ }^{26}$

\begin{tabular}{lccccccccc}
\hline$\%$ & DK & FR & UK & ITA & NL & PO & ES & BE & UE \\
\hline Nacional & 85.3 & 98.0 & 85.2 & 32.1 & 68.4 & 93.3 & 29.1 & 24.7 & 66.2 \\
Subnacional & 14.7 & 2.0 & 14.8 & 67.9 & 31.6 & 6.7 & 70.9 & 75.3 & 33.8 \\
\hline
\end{tabular}

Sin embargo, las diferencias regionales en España son indiscutibles y se han ido pronunciando a lo largo de los últimos ańos, pues aquellas regiones que lo necesitaban no han potenciado el gasto industrial con respecto al peso de su economía. 
Además del presupuesto industrial y del peso que supone el sector secundario en la estructura regional, los indicadores de Gasto en I+D ejecutado por el sector empresarial privado
(GIDE) y el Gasto en Innovación (GINN) reflejan estas diferencias.

Tabla 5. Distribución de los recursos dedicados por las empresas a innovación tecnológica e I+D por CCAA. Porcentaje GIDE/GINN26

\begin{tabular}{lccccccccccc}
\hline Total & MAD & CAT & EUS & AND & CVA & GAL & C-LE & ARA & NAV & C-MA & RESTO \\
\hline 39 & 39 & 45.5 & 52 & 26.5 & 31.5 & 25.8 & 45.8 & 22.2 & 65.3 & 22.1 & 30.9 \\
\hline
\end{tabular}

Pocas cosas han cambiado desde que en 2005 el 50\% del gasto en innovación correspondiese a Madrid y Barcelona, que junto con el País Vasco suponían el 70\% del gasto ejecutado. Esto significaba que, a excepción de estas tres regiones, en nuestro país no se renovaban los productos/servicios con la necesaria velocidad y se invertía poco en investigación. Con el paso de los años las empresas comprendieron que su capacidad de aprendizaje dependía de sus trabajadores y que había que buscar un equilibrio entre la compra y el desarrollo interno de conocimientos para seguir innovando. En empresas de alta tecnología o grandes empresas de sectores de tecnología media-baja (manufactura y servicios), hace una década la actividad que más recursos consumía era la $\mathrm{I}+\mathrm{D}$ interna, mientras que la adquisición de equipos era la más importante en empresas de sectores de tecnología medio-alta, construcción y empresas manufactureras de tecnología medio-baja ${ }^{27}$.

Para reconocer en una región periférica las características de su sistema de innovación hay que fijarse en primer lugar en la disponibilidad, composición y distribución económica, humana y de actividades en I+D. Si observamos mucho esfuerzo empresarial e intensa conexión de entidades públicas de investigación con las empresas la situación de competitividad en innovación será fuerte. En los sistemas maduros y equilibrados el esfuerzo se desplaza hacia el lado empresarial. En segundo lugar nos fijaremos en la capacidad de absorción o competencia para identificar, asimilar y explotar los conocimientos científicos y tecnológicos. Esta capacidad depende del tamaño de las empresas, la estructura sectorial y la formación de los trabajadores. En la mayor parte de regiones periféricas españolas abundan las pequeñas y medianas empresas, lo que implica una menor capacidad para desarrollar actividades de I+D por sí mismas. En último lugar, es importante analizar cómo se articulan las relaciones entre las empresas y los demás ayudantes del sistema para buscar sinergias y complementariedades. Hace una década nuestro país tenía uno de los índices de cooperación empresarial en innovación más bajos, y aunque no se han conseguido avanzar demasiado, en los últimos años la colaboración empresarial ha ido aumentando progresivamente. En el sector servicios, el más identificado con el deporte, cuanto mayor es el tamańo de las empresas, más cooperación ${ }^{28}$.

De acuerdo con los criterios de la $\mathrm{OCDE}^{1}$, regiones como Galicia por ejemplo, tienen muy poco peso el empleo en las manufacturas de alta tecnología $(0,2 \%)$ y en los servicios de alta tecnología $(1,6 \%)$. Igualmente las manufacturas de tecnología media-alta apenas representan el 5,2\% del empleo total, las más importantes relacionadas con el automóvil. Tanto si nos referimos al número de patentes solicitadas a la Oficina de Patentes en España como en Europa, Galicia está considerablemente por debajo de la media española y muy lejos de la media comunitaria. El gasto en $\mathrm{I}+\mathrm{D}$ ha subido y bajado en igual proporción en la última década, con un pico en 2008 cuando se invirtieron 584 millones $€$ a través de las universidades (38,6\%), centros públicos (13,3\%), y empresas $(48,1 \%)$. $\mathrm{Si}$ bien el gasto empresarial ha subido ligeramente en estos años, todavía está por debajo de la media comunitaria, y muy condicionada con la inyección de recursos públicos en centros tecnológicos, lo que desvirtúa el análisis. Además tanto el peso de los profesionales en $\mathrm{I}+\mathrm{D}$ en las empresas como en las universidades es muy bajo, y maneja proporcionalmente recursos inferiores a los de sus homólogos europeos, aunque su número sea mayor ${ }^{29}$.

La situación gallega es similar a la mayoría de regiones de nuestro país. En el índice RIS-2009 la sitúa en el grupo de regiones medio-bajo innovadoras, junto a una gran parte de territorios españoles y portugueses, además de algunos italianos $^{30}$. Los países menos innovadores son aquellos que tienen una mayor concentración territorial de innovación en un menor número de regiones, un mapa atomizado, como en nuestro caso. En cualquier sector existen innovaciones y necesidad de innovar, pero es cierto que teniendo una estructura económica dominada por sectores de baja o media-baja intensidad tecnológica es complicado encontrar una palanca pro-innovación en el tejido empresarial.

En España, además la alta concentración observada contrasta con la elevada descentralización administrativa y política, entre las mayores de Europa, lo que implica una mayor necesidad de coordinación entre el Estado y las Comunidades Autónomas, que no está produciendo plenamente. Mientras esta situación se demore la pérdida de recursos será evidente. En diversas ocasiones, las soluciones para la apoyar la innovación han sido los auténticos problemas. En primer lugar, o hay que reflexionar más sobre ellos o hemos de olvidarnos de los modelos ideales, puesto en nuestro país se han copiado repetidamente casos de éxito de regiones más avanzadas con estructuras productivas y sociales diferentes, sin considerar sus 
características autóctonas o el hándicap que suponía nuestro bajo límite regional de esfuerzo en actividades de investigación y tecnología, además de la escasa capacidad de absorción que tiene nuestro entorno productivo territorial. Para acercarnos al potencial innovador de una región no es suficiente con hacer ciencia, absolutamente necesaria, sino que habrá que considerar además que la dinámica territorial está condicionada por su estructura productiva, los sectores predominantes, el tipo de empresas, la implicación del sistema financiero, y la regulación pública.

En el sector deportivo para el futuro será necesario iden- tificar con precisión a los protagonistas nacionales y regionales involucrados e impulsar organismos facilitadores que permitan la puesta en común de conocimiento, experiencias y refuerzo de las políticas públicas. Es prioritario esforzarse en crear redes multidisciplinares región-nación, a la vez que incorporar titulados universitarios a las empresas que realicen allí sus proyectos formativos, fomentar el espíritu creativo desde la escuela y el tejido industrial, y apoyar a las empresas más intensas en el conocimiento e investigación, que con seguridad serán las más atractivas para los inversores.

\section{Referencias}

1. Organización para la Cooperación y Desarrollo Económicos (2013). Strategic orientations of the secretary-general 2013 and beyond [citado 21 Nov 2014]. Disponible en: http://www.oecd.org/mcm/CMIN(2013)1-ENG.pdf

2. Unión Europea (2013). Council conclusions on the contribution of sport to the EU economy, and in particular to addressing youth employment and social inclusion. [citado 03 Dic 2014]. Disponible en: http://www.consilium.europa.eu/uedocs/cms_data/docs/pressdata/en/ educ/139733.pdf

3. SpEA-SportEconAustria (Leader), SIRC-Sport Industry Research Centre, SSRC-Statistical Service of the Republic of Cyprus, MSPMeerwaarde Sport en Economie, FESI-Federation of the European Sporting Goods Industry, \& MSTRP-Ministry of Sport and Tourism of the Republic of Polan (2012). Study on the contribution of sport to economic growth and employment in the EU. [citado 20 Nov 2014]. Disponible en: http://ec.europa.eu/sport/library/studies/study-contribution-spors-economicgrowth-final-rpt.pdf

4. Vocasport (2004). Vocational Education in Training in the Field of Sport in the European Union: Situation, Trends and Outlook. [citado 12 Nov 2014]. Disponible en: http://www.kirolan.org/El\%20sector\%20del\%20empleo\%20deportivo/0.1.1\%20lotvocasport_en.pdf.

5. Andreff, W., \& Szymanski, S. (2006). Handbook of the Economics of Sport. Cheltenham, Northampton: Edward Elgar.

6. Benítez, J.J., Lacomba, B (2012). Los avances en la valoración económica del deporte en Europa. Estudios de Economía Aplicada. [citado 11 Oct 2013]; 30 (2): 637-654. Disponible en: http://www.redalyc.org/ articulo.oa?id=30124481013

7. Unión Europea (2014). Estudio sobre la contribución del deporte al crecimiento económico y el empleo en la UE. [citado 02 Dic 2014]. Disponible en: http://ec.europa.eu/sport/library/studies/study-contribution-spors-economic growth-final-rpt.pdf

8. Hewings, G., \& Jensen, C. (1986). Regional, Interregional and Multiregional Input-Output Analysis. En Nijkon P, Handbook of Regional and Urban Economics. (pp. 295-355). Vol 1. Amsterdam: North Holland.

9. Miller, R.E., Blair, P.D. (1985). Input-Output-Analysis: Foundations and Extensions. New Jersey. Prentice- Hall.

10. Unión Europea (2014). Sport keeps not only you, but also industry fit. [citado 23 Nov 2014]. Disponible en: http://europa.eu/rapid/press-release_MEMO-1435_en.htm

11. Euroreporter (2014). Sport as a growth engine for EU economy. [citado 10 Dic 2014].Disponible en: http://www.eureporter.co/es/magazine/2014/06/19/sportas-a-growth-engine-for-eu-economy/

12. Market Psych Data (2014). The Bluefin Solutions Elastic Innovation Index. Global top 50 most innovati- ve companies. [citado 9 Nov 2014]. Disponible en: http://www.bluefinsolutions.com/Bluefin/media/Bluefin/PDFS/BluefinSolutionsElastic-Innovation-Index-2014-Global-top-50-most-innovative-companies(2).pdf.

13. Fast Company Staff (2014). The most innovate companies 2013. [citado 18 Sep 2014]. Disponible en: http://www.fastcompany.com/mostinnovative-companies/2013/full-list.

14. Kate Rockwood (2014). The most innovate companies 2013 in fitness. [citado 19 Sep 2014]. Disponible en: http://www.fastcompany.com/ most-innovative-companies/2013/industry/fitness.

15. Innosport (2014). Innosport Platform [citado 20 Dic 2014]. Disponible en: http://www.government.nl/issues/sports/research-and-innovationin-sport

16. Profit Project (2008). Profit Project. [citado 24 Nov 2014]. Disponible en: http://www.fieldlabs.eu/about-main/about-profit

17. Unión Europea (2014). Education, Youth, Culture and Sport. [citado 22 Nov 2014]. Disponible en: http://www.consilium.europa.eu/uedocs/cms_data/docs/pressdata/en/ educ/14204.pdf

18. European Commission (2014). Communication from the Commission to the European Parliament, the Council, the European Economic and Social Committe and the Committe of the Regions. A budget for Europe 2020. [citado 11 Dic 2014] Disponible en: http://ec.europa.eu/budget/library/bibliodocuments/fin_fwk1/420/ MFF_COM2011-500_Part_I_en.pdf

19. Unión Europea (2014). What is COSME? [citado 28 Nov 2014]. Disponible en: http://ec.europa.eu/enterprise/initiatives/cosme/index_ en.htm

20. Diario Oficial de la Unión Europea (2014). Conclusiones del Consejo y de los representantes de los gobiernos de los Estados Miembros, reunidos en el seno del Consejo, sobre el deporte como motor de la innovación y del crecimiento económico (2014/C 436/02). 5 de diciembre de 2014. Disponible en: http://eur-lex.europa.eu/legalcontent/ES/TXT/ PDF/?uri=OJ:C:2014:436:FULL\&from=ES

21. Ministerio de Economía y Competitividad (2014). Programa Horizonte 2020. [citado 25 Nov 2014]. Disponible en: http://www.eshorizonte2020.es/

22. Nieto, A. (2003). La investigación en el marco constitucional: las OPIS, el CSIC y las Comunidades Autónomas. Arbor: Ciencia, pensamiento y cultural. 695-696: 1-18.

23. Unión Europea (2014). Horizon 2020. The EU Framework Programme for Research and Innovation. [citado 25 Nov 2014]. Disponible en: http://ec.europa.eu/programmes/horizon2020/en/h2020-section/ leadershipenabling-and-industrial-technologies

24. Fernández, I., Castro, E., \& Zabala, J.M . (2006): Estrategias regionales de innovación: el caso de las regiones europeas periféricas. En: Vence, 
X. (Coord), Crecimiento y políticas de innovación. Nuevas tendencias y experiencias comparadas. (pp. 157-191). Madrid: Ediciones Pirámide.

25. Mas-Verdú, F. (2005). Política industrial y sectores tradicionales, Economía industrial. Madrid. Ministerio de Industria, Comercio y Turismo. 355-356 (pp. 47-58).

26. Instituto Nacional de Estadística (2006). Encuesta de Innovación Tecnológica en Empresas 2006 [citado 10 Oct 2014]. Disponible en: http://www.ine.es/jaxi/menu.do?type=pcaxis \&file=pcaxis $\&$ path= $\% 2 \mathrm{~F}$ $\mathrm{t} 14 \% 2 \mathrm{Fp} 01 \% 2 \mathrm{~F} \% 2 \mathrm{Fa} 2006$

27. Instituto Nacional de Estadística (2004). Estrategia de innovación de los sectores en función de la distribución del gasto en innovación tecnológica en 2004. [citado 10 Oct 2014]. Disponible en: http://www.ine.es/jaxi/menu.do?type=pcaxis $\&$ file $=$ pcaxis $\&$ path $=\% 2 \mathrm{~F}$ t14\%2Fp01\%2F\%2Fa2004
28. Instituto Nacional de Estadística (2012). Estadísticas de Innovación Tecnológicas en las empresas 1998-2012. [citado 13 Oct 2014]. Disponible en: http://www.ine.es/jaxi/menu.do?type=pcaxis \&path=\%2Ft14\%2Fp061 \&file $=$ inebse $\& \mathrm{~L}=0$

29. Vence, X. (2010). Sistema Galego de Innovación e Políticas de Innovación: Balance dunha década. Revista Galega de Economía. 19 (extra): 161-190.

30. Hollanders, H., Tarantola, S., Loschky, A. (2009). Regional Innovation Scoreboard 2009. Pro-Inno Europe, Innometrics. [citado 20 Nov 2014]. Disponible en: http://ec.europa.eu/enterprise/policies/innovation/files/ ris-2009_en.pdf 